Copyright (C) 2021 University of Bucharest Printed in Romania. All rights reserved

ISSN print: $1224-5984$

ISSN online: $2248-3942$
Rom Biotechnol Lett. 2021; 26(6): 3047-3053 doi: $10.25083 / \mathrm{rbl} / 26.6 / 3047-3053$

Received for publication, August, 29, 2020

Accepted, November, 30, 2020

Original paper

\title{
Online Advanced Bacterial Identification Software, an Original Tool for Phenotypic Bacterial Identification
}

\author{
IONUT SORESCU ${ }^{1}$, COSTIN STOICA $^{2}$ \\ ${ }^{I}$ National Research Development Institute for Animal Biology and Nutrition (IBNA), Biotechnology \\ Laboratory, 1 Calea Bucuresti, Balotesti, Ilfov, 077015, Romania; Present address: Institute for \\ Diagnosis and Animal Health, Bacteriology Laboratory, 63 Dr. Staicovici, 050557 Bucharest, Romania. \\ ${ }^{2}$ Romvac Company, 7 Soseaua Centurii, Voluntari 077190, Ilfov, Romania
}

\begin{abstract}
The objective of the study is to present and validate an original online Advanced Bacterial Identification Software, ABIS, by comparison to a commercially available, standardized identification system, API strips and apiweb ${ }^{\mathrm{TM}}$ bioMerieux software.

Methods and results: presentation of ABIS online software, phenotypic bacterial identification of 16 reference strains and 123 wild isolates by ABIS and apiweb TM bioMerieux software and comparative analysis of results. Closed results were obtained (same taxa) for reference and wild strains of Enterobacteriaceae, Pasteurellaceae, Bacillaceae, Lactobacillaceae, Staphylococcaceae, Streptococcaceae, and other.

Conclusions: Apiweb ${ }^{\mathrm{TM}}$ confirmed the results of ABIS, overall, average identification percent for ABIS being $91.8 \%$ and $90.4 \%$ for apiweb TM. ABIS online is a powerful tool for microbiology lab and the Encyclopedia connection provides essential information about the ecological significance, pathology and other features of the identified strains.
\end{abstract}

Keywords phenotypic bacterial identification, original online software

To cite this article: SORESCU I, STOICA C. Online Advanced Bacterial Identification Software, an Original Tool for Phenotypic Bacterial Identification. Rom Biotechnol Lett. 2021; 26(6): 3047-3053. DOI: $10.25083 / \mathrm{rbl} / 26.6 / 3047-3053$.

*Corresponding author: IONUT SORESCU, National Research Development Institute for Animal Biology and Nutrition (IBNA), Biotechnology Laboratory, 1 Calea Bucuresti, Balotesti, Ilfov, 077015, Romania; +40749151806.

E-mail: ionutsorescu68@gmail.com 


\section{Introduction}

In the fields of applied bacteriology, human and veterinary clinic, phytopathology and also in environmental microbiology, bacterial strains from various sources are routinely isolated and phenotypically identified. Beside a properly equipped laboratory, this activity, on the edge between science, technique, craftsmanship and art, requires specific culture media, reagents, experienced specialists in bacteriology, and a bacterial identification system. The identification systems may be the manuals of bacteriology (Borriello, 2006; Buiuc, 2009; Gordon, 1973; Dworkin, 2006), Bergey's Manuals of Determinative (Buchanan, 1975; Holt, 1994) and Systematic (Vos, 2009; Goodfellow, 2012; Garitty, 2004) Bacteriology, review articles on specific bacteria categories (Funke, 1997), commercial systems like apiweb ${ }^{\text {TM }}$ (bioMerieux), Biolog, and online systems. Each of these systems has its limitations, well-known by the specialists (no interactivity, no updating for the newly described taxa, constraining the user to a predefined set of tests, no instant connection to a database of ecological significance and pathology of the isolated strains). To tackle these problems we created ABIS online - Advanced Bacterial Identification Software (http://www.tgw1916.net), a laboratory tool for bacterial identification, based on morphology, biochemical characters, cultural characteristics, ecology and pathogenicity data. The program allows a great flexibility in choosing biochemical tests and it is an alternative to commercial systems, code-books or identification tables. It is a free software, available on World Wide Web since 2007, being increasingly used by students, young researches, biologists and human and veterinary clinicians.

The aim of the study was to present and validate ABIS software by comparison to a commercially available, standardized identification system.

\section{Materials and Methods}

ABIS online (ABIS online - bacterial identification software version 12, http://www.tgw1916.net) is a free bacterial identification software available on World Wide Web.

The identification scheme is based on the comparison between the morphological and biochemical character values of the isolated strain and standard values of taxa contained in the database. Thirteen databases are currently available, covering areas of Enterobacteriaceae, Pasteurellaceae, Campylobacteraceae, Bacillaceae, Lactobacillaceae, Staphylococcaceae, Streptococcaceae, Clostridium, Vibrio and Aeromonas, Listeriaceae, Neisseriaceae, Chromobacterium, Corynebacterium, and Non-fermenters (fig. 1).

The databases were created taking into consideration the characteristics of type strains of each species, the characteristics of strains existing in international collections and rigorously characterized strains, generally described in Bergey's Manual of Systematic Bacteriology (7-9), Bergey's Manual of Determinative Bacteriology (5, 6), International Journal of Systematic and Evolutionary Microbiology, Topley \& Wilson's Manual (1), as well as review articles (10).

ABIS is not an annex of an identification kit, so the type and number of the required tests are not limited by a pattern and can vary by lab availabilities (fig. 2). Depending on the number of the introduced tests, ABIS calculates the percentage of similarity with taxa from the database and the accuracy of identification (fig. 3). The quality of results depends on the number of tests entered by the user. At least 8 tests are required, and the maximum depends on laboratory capacity and database limitations. ABIS databases contain an average of 40-50 tests from which the user can choose.

The most important feature of ABIS is that it allows the user to instantly access data from Encyclopedia about morpho-biochemical characters, cultural characteristics, ecology and pathogenicity of the identified taxa, leaving the final decision to the human factor, not to the machine.

The morphological and biochemical characters of 16 reference strains and 123 wild isolates were tested by ABIS online and apiweb ${ }^{\mathrm{TM}}$ software (bioMerieux) using all laboratory available tests (classical tests and API bioMerieux strips). The reference strains were derived from ROMVAC Company and Cantacuzino Institute Bacterial Collections. The wild strains were isolated from various human, animal and environmental sources: milk, urine, pus, vaginal secretion, ear secretion, skin, internal organs from deceased animals (liver, kidney, lungs, bone marrow, salpinx), yolk sac, sanitation tests and animal feed.

Commercially available tests (Gram staining, oxidase, Simmons citrate, Triple Sugars Iron -TSI-, Motility Indole Urea -MIU-, hemolysis on sheep blood agar), together with commercially available API bioMerieux kits (API 20E, API 20 NE, API Staph, API Strep, API Campy, API 20A and API 50CHB/CHL) were used for identification.

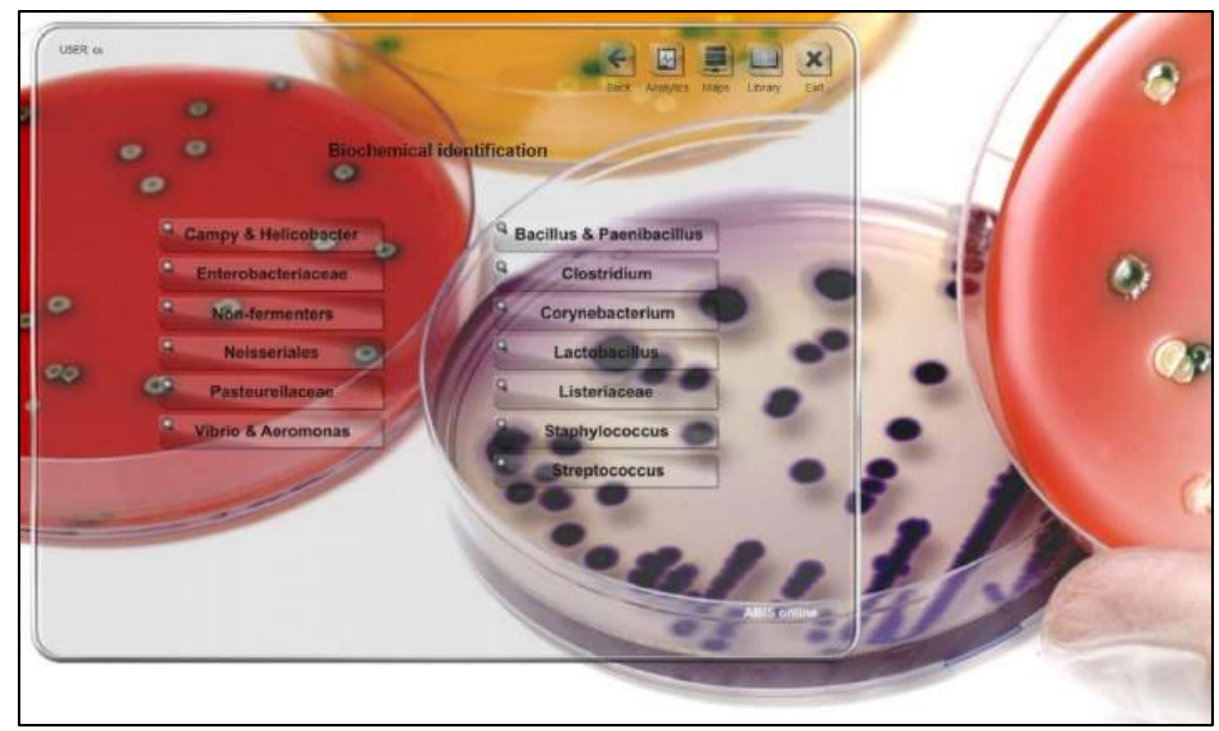

Figure 1. ABIS online Main Menu - Selecting databases. 


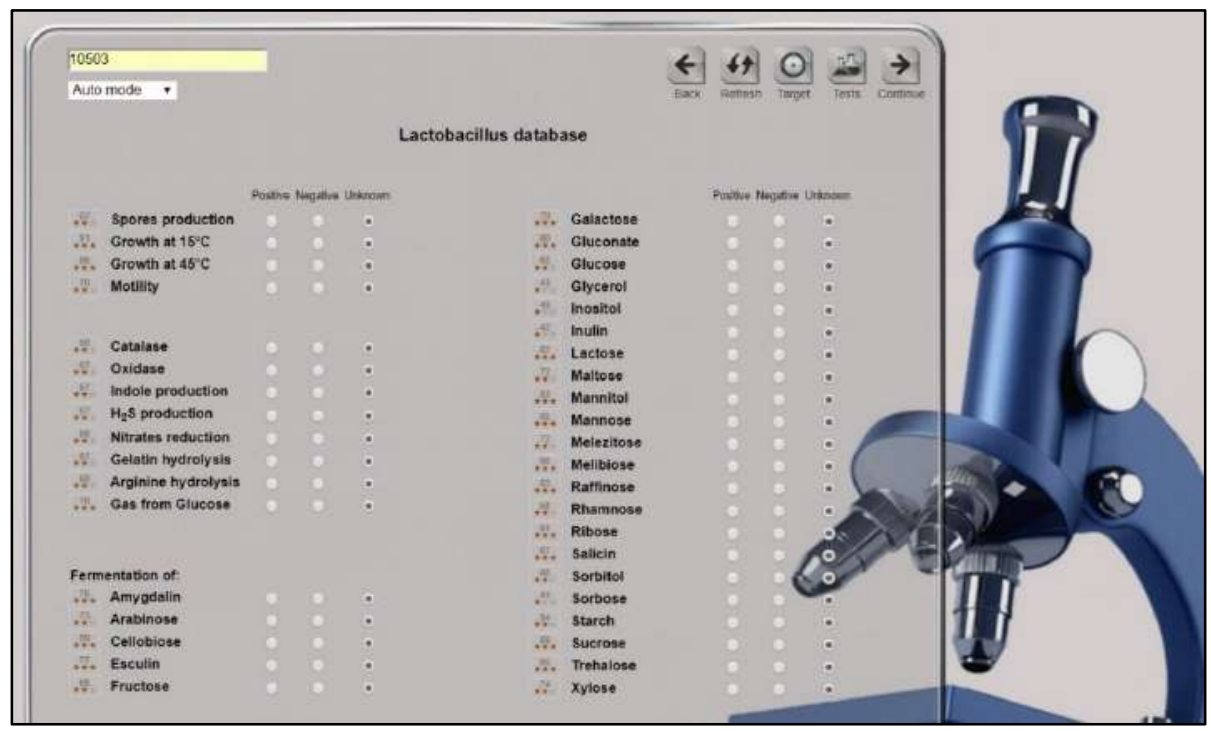

Figure 2. Input page of ABIS online.

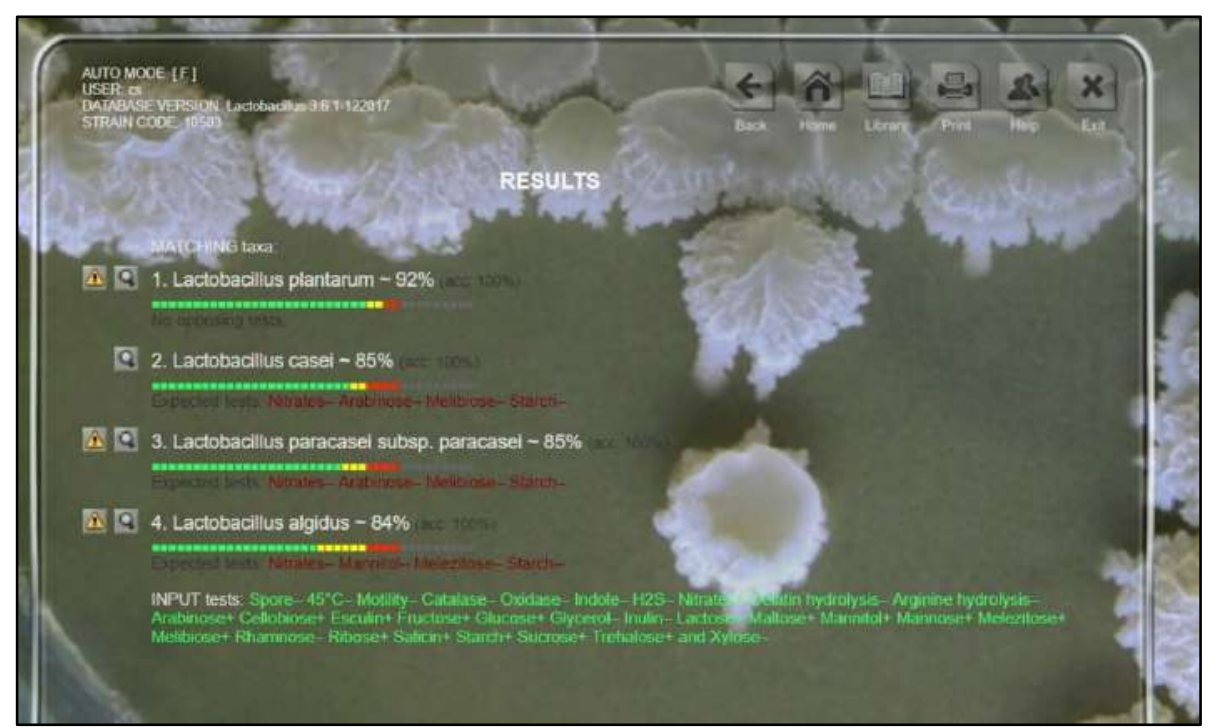

Figure 3. Results page of ABIS online.

\section{Results and discussion}

Table 1 shows the tested reference strains and results of comparative identification with ABIS online and apiweb ${ }^{\mathrm{TM}}$ software.

Very closed results (same taxa, with low difference, $\leq$ $10 \%$, in $\mathrm{id} \%$ ) were obtained for reference strains in comparative identification with ABIS online and apiweb ${ }^{\mathrm{TM}}$ software.

Table 2 shows the tested wild strains together with their source of isolation and results of comparative identification with ABIS online and apiweb ${ }^{\mathrm{TM}}$ software.

Comparable results (same taxa), but with important difference in the average $\mathrm{id} \%$ for Staphylococcus spp. $(\leq 48.3 \%)$, Streptococcus spp. $(\leq 29 \%)$ and Stenotrophomonas maltophilia (46\%), were obtained for wild strains in comparative identification with ABIS online and apiweb ${ }^{\mathrm{TM}}$ software.

Both software programs returned the same taxa with a close identification percent for reference strains. For the Clostridium tetani RO-2009 strain, apiweb ${ }^{\mathrm{TM}}$ returned Clostridium tetani identity without an id \%.

Comparable results were obtained in ABIS and apiweb ${ }^{\mathrm{TM}}$ bacterial identification systems, both for reference and wild strains. In most cases both software programs returned the same taxa and a close identification percent.

For 16 strains apiweb ${ }^{\mathrm{TM}}$ considered the profile to be "unacceptable", thus returned a suggested taxon wihout calculating the $\mathrm{id} \%$ and without validating its results. In all these cases ABIS confirmed apiweb's suggested taxa with a high id $\%$. 
Table 1. Comparative reference strains identification between ABIS online and apiweb ${ }^{\mathrm{TM}}$ software

\begin{tabular}{|c|c|c|c|}
\hline \# & Strain name and code & ABIS results id $\%^{a}$ & apiweb $^{\mathrm{TM}}$ results id $\% \mathrm{o}^{\mathrm{b}}$ \\
\hline 1. & $\begin{array}{l}\text { Staphylococcus aureus } \\
\text { ATCC } 25293\end{array}$ & $\begin{array}{l}\text { Staphylococcus aureus subsp. } \\
\text { aureus } 99 \%\end{array}$ & Staphylococcus aureus $97.8 \%$ \\
\hline 2. & $\begin{array}{l}\text { Helicobacter pylori } \\
\text { ATCC } 43504\end{array}$ & Helicobacter pylori $94 \%$ & Helicobacter pylori $99.9 \%$ \\
\hline 3. & $\begin{array}{l}\text { Bacillus thuringiensis } \\
\text { NCIMB } 9134\end{array}$ & Bacillus thuringiensis $93 \%$ & $\begin{array}{l}\text { Bacillus cereus, possibility of } \\
\text { B. thuringiensis } 98.9 \% \\
\end{array}$ \\
\hline 4. & $\begin{array}{l}\text { Bacillus megaterium } \\
\text { NCIMB } 8508\end{array}$ & Bacillus megaterium 90\% & Bacillus megaterium $99.6 \%$ \\
\hline 5. & $\begin{array}{l}\text { Clostridium tetani } \\
\text { RO-2009 }\end{array}$ & Clostridium tetani $97 \%$ & Clostridium tetani (no id\%) \\
\hline 6. & $\begin{array}{l}\text { Salmonella Choleraesuis } \\
\mathrm{RO}-014\end{array}$ & Salmonella spp. 87\% & Salmonella choleraesuis $99.7 \%$ \\
\hline 7. & $\begin{array}{l}\text { Salmonella Typhimurium } \\
\text { ATCC } 14028\end{array}$ & Salmonella spp. 94\% & Salmonella spp. 99.9\% \\
\hline 8. & $\begin{array}{l}\text { Salmonella Typhimurium } \\
\text { RO-TM2 }\end{array}$ & Salmonella spp. 89\% & Salmonella spp. 99.0\% \\
\hline 9. & $\begin{array}{l}\text { Proteus hauseri } \\
\text { ATCC } 13315\end{array}$ & Proteus vulgaris / P. hauseri $95 \%$ & Proteus vulgaris group $99.8 \%$ \\
\hline 10. & $\begin{array}{l}\text { Escherichia coli } \\
\text { ATCC } 8739\end{array}$ & Escherichia coli $98 \%$ & Escherichia coli $99.9 \%$ \\
\hline 11. & $\begin{array}{l}\text { Streptococcus mutans } \\
\text { ATCC } 55677\end{array}$ & Streptococcus mutans $98 \%$ & Streptococcus mutans $99.9 \%$ \\
\hline 12. & $\begin{array}{l}\text { Enterococcus faecium } \\
\text { NCIMB } 10415 \\
\end{array}$ & Enterococcus faecium $97 \%$ & Enterococcus faecium $93.9 \%$ \\
\hline 13. & $\begin{array}{l}\text { Enterococcus faecium } \\
\text { NCIMB } 11181\end{array}$ & Enterococcus faecium $92 \%$ & Enterococcus faecium $99.2 \%$ \\
\hline 14. & $\begin{array}{l}\text { Pseudomonas aeruginosa } \\
\text { ATCC } 15442\end{array}$ & Pseudomonas aeruginosa $92 \%$ & Pseudomonas aeruginosa $98.1 \%$ \\
\hline 15. & $\begin{array}{l}\text { Lactobacillus plantarum } \\
\text { ATCC } 8014\end{array}$ & Lactobacillus plantarum 91\% & Lactobacillus plantarum $199.9 \%$ \\
\hline 16. & $\begin{array}{l}\text { Lactobacillus paracasei } \\
\text { CCM } 1837\end{array}$ & $\begin{array}{l}\text { Lactobacillus paracasei subsp. } \\
\text { paracasei } 94 \%\end{array}$ & $\begin{array}{l}\text { Lactobacillus paracasei subsp. } \\
\text { paracasei } 1 / 3 \\
48.0 / 51.0 \%\end{array}$ \\
\hline
\end{tabular}

Legend: a- ABIS \%id represents the percentage of similarity with taxa from the database, all databases containing a matrix where probabilistic incidence values are allocated for every taxon and their corresponding morpho-biochemical characters; b- apiweb ${ }^{\mathrm{TM}} \% \mathrm{id}$ is a probabilistic calculation using bioMerieux own system procedure.

Table 2. Comparative wild strains identification between ABIS online and apiweb ${ }^{\mathrm{TM}}$ software.

\begin{tabular}{|c|c|c|c|c|}
\hline \# & Source & $\begin{array}{l}\text { Nr. of } \\
\text { tested } \\
\text { strains }\end{array}$ & $\begin{array}{l}\text { ABIS results } \\
\text { Average id } \% \text { a }\end{array}$ & $\begin{array}{l}\text { apiweb }^{\mathrm{TM}} \text { results } \\
\text { Average id\% } \%\end{array}$ \\
\hline 1 . & $\begin{array}{l}\text { Goat mastitis, chicken } \\
\text { arthritis, dog skin, cat } \\
\text { skin, cow milk, chicken } \\
\text { samples }\end{array}$ & 11 & Staphylococcus aureus $94.9 \%$ & Staphylococcus aureus $94.8 \%$ \\
\hline 2. & Cow milk & 1 & Staphylococcus chromogenes $93 \%$ & Staphylococcus chromogenes $86.7 \%$ \\
\hline 3. & $\begin{array}{l}\text { Sheep and cow milk, } \\
\text { vaccine contaminant }\end{array}$ & 4 & Staphylococcus epidermidis $96 \%$ & Staphylococcus epidermidis $94.4 \%$ \\
\hline 4. & Dog and cat skin & 4 & Staphylococcus intermedius $96 \%$ & Staphylococcus intermedius $62.1 \%$ \\
\hline 5. & Dog skin & 2 & Staphylococcus haemolyticus $91 \%$ & Staphylococcus haemolyticus $62 \%$ \\
\hline & Dog otitis & 1 & Staphylococcus schleiferi $93 \%$ & Staphylococcus schleiferi $44.7 \%$ \\
\hline & Dog skin & 1 & Staphylococcus simulans $89 \%$ & Staphylococcus simulans $96.0 \%$ \\
\hline
\end{tabular}


Online Advanced Bacterial Identification Software

\begin{tabular}{|c|c|c|c|}
\hline 8. Goat and cow milk & 3 & Staphylococcus xylosus 95\% & Staphylococcus xylosus $98.4 \%$ \\
\hline 9. $\quad$ Environment & 1 & Staphylococcus carnosus $88 \%$ & Staphylococcus carnosus (no id\%) \\
\hline $\begin{array}{l}10 \text { Human skin lesion pus, } \\
\text { goat and sheep milk }\end{array}$ & 3 & Streptococcus agalactiae $92.6 \%$ & Streptococcus agalactiae $99.7 \%$ \\
\hline 11 Sheep intestine & 1 & Streptococcus bovis $96 \%$ & Streptococcus bovis $67 \%$ \\
\hline 12 Swine lungs & 2 & $\begin{array}{l}\text { Streptococcus dysgalactiae subsp. } \\
\text { equisimilis } 84 \%\end{array}$ & $\begin{array}{l}\text { Streptococcus dysgalactiae subsp. } \\
\text { equisimilis } 92.5 \%\end{array}$ \\
\hline 13 Goat milk & 1 & Streptococcus mutans $83 \%$ & Streptococcus mutans $99.7 \%$ \\
\hline 14 Rabbit lungs, human throat & 2 & $\begin{array}{l}\text { Streptococcus pyogenes } 88 \% \\
\text { (alternative: Gemella palaticanis } 86 \% \text { ) }\end{array}$ & $\begin{array}{l}\text { Streptococcus pyogenes } 43.9 \% \\
\text { (alternative: } \text { S. oralis } 88.3 \% \text { ) }\end{array}$ \\
\hline 15 Pig lungs, pig arthritis & 2 & Streptococcus suis $90 \%$ & Streptococcus suis $98.8 \%$ \\
\hline 16 Bovine vaginal secretion & 1 & Enterococcus durans $98 \%$ & Enterococcus durans $99.4 \%$ \\
\hline 17 Chicken samples, dog skin & 4 & Enterococcus faecalis $93 \%$ & Enterococcus faecalis $99.1 \%$ \\
\hline $\begin{array}{l}18 \text { Bovine vaginal secretion, } \\
\text { goat kidney }\end{array}$ & 2 & Enterococcus faecium $92 \%$ & Enterococcus faecium $95.7 \%$ \\
\hline 19 Cow milk & 1 & Lactococcus lactis, subsp. Lactis $90 \%$ & Lactococcus lactis, subsp. lactis $90.4 \%$ \\
\hline $\begin{array}{l}20 \text { Sanitation test from swine } \\
\text { farm }\end{array}$ & 1 & Stenotrophomonas maltophilia $91 \%$ & Stenotrophomonas maltophilia $45 \%$ \\
\hline 21 Chicken yolk sac, water & 2 & Pseudomonas aeruginosa $88.5 \%$ & Pseudomonas aeruginosa $98.8 \%$ \\
\hline $\begin{array}{l}22 \text { Dog urinary infection, cat } \\
\text { vaginal secretion }\end{array}$ & 2 & Chryseomonas luteola 91\% & $\begin{array}{l}\text { Chryseomonas luteola } 99.8 \% \\
\text { (no id\% for } 1 \text { strain) }\end{array}$ \\
\hline 23 Chicken feces & 1 & Moraxella lacunata $96 \%$ & Moraxella lacunata $85.7 \%$ \\
\hline 24 Chicken yolk sac & 1 & $\begin{array}{l}\text { Acinetobacter baumannii / } A \text {. } \\
\text { calcoaceticus } 91 \%\end{array}$ & $\begin{array}{l}\text { Acinetobacter baumannii / } A \text {. } \\
\text { calcoaceticus } 91 \%\end{array}$ \\
\hline 25 Chicken intestine & 1 & $\begin{array}{l}\text { Campylobacter jejuni subsp. jejuni } \\
\text { biotype } 186 \%\end{array}$ & $\begin{array}{l}\text { Campylobacter jejuni subsp. jejuni } \\
\text { biotype } 199.4 \%\end{array}$ \\
\hline $\begin{array}{l}26 \text { Chicken salpinx, guinea } \\
\text { pig lungs }\end{array}$ & 2 & $\begin{array}{l}\text { Mannheimia haemolytica } 85 \% \\
\text { (alternative: } \text { M. glucosida } 77 \%-1 \\
\text { strain) }\end{array}$ & $\begin{array}{l}\text { Mannheimia haemolytica / } \\
\text { Pasteurella trehalosi } 77.5 \%\end{array}$ \\
\hline $\begin{array}{l}27 \text { Swine samples, chicken } \\
\text { liver and bone marrow }\end{array}$ & 4 & Pasteurella multocida 89\% & Pasteurella multocida $95.0 \%$ \\
\hline 28 Goat vaginal secretion & 1 & Vibrio parahaemolyticus $77 \%$ & Vibrio parahaemolyticus $98.9 \%$ \\
\hline 29 Guinea pig intestine and liver & 2 & Aeromonas hydrophila $86 \%$ & Aeromonas hydrophila $97.9 \%$ \\
\hline 30 Cow milk & 1 & Bacillus licheniformis $85 \%$ & Bacillus licheniformis $99.9 \%$ \\
\hline 31 Cow milk & 1 & Bacillus pumilus $80 \%$ & Bacillus pumilus $99.9 \%$ \\
\hline 32 Pig and chicken intestine & 3 & Lactobacillus fermentum $89.6 \%$ & Lactobacillus fermentum $71.7 \%$ \\
\hline 33 Bovine intestine & 1 & Lactobacillus rhamnosus $84 \%$ & Lactobacillus rhamnosus $99.9 \%$ \\
\hline 34 Bovine intestine & 1 & $\begin{array}{l}\text { Lactobacillus paracasei subsp. } \\
\text { paracasei } 98 \%\end{array}$ & $\begin{array}{l}\text { Lactobacillus paracasei subsp. } \\
\text { paracasei } 98 \%\end{array}$ \\
\hline $\begin{array}{l}35 \text { Vaccine and medical } \\
\text { product contaminants }\end{array}$ & 3 & Citrobacter freundii $96.5 \%$ & Citrobacter freundii $99.8 \%$ \\
\hline 36 Chicken feces & 3 & Citrobacter youngae $91.6 \%$ & Citrobacter youngae $99.6 \%$ \\
\hline 37 Dog conjunctivitis & 1 & Enterobacter amnigenus biotype II $96 \%$ & Enterobacter amnigenus biotype II 96\% \\
\hline $\begin{array}{l}38 \text { Chicken samples, animal } \\
\text { feed, cow milk, dog otitis }\end{array}$ & 8 & Enterobacter cloacae $94.7 \%$ & Enterobacter cloacae $95.2 \%$ \\
\hline $\begin{array}{l}39 \text { Chicken bone marrow (2) } \\
\text { and yolk sac }\end{array}$ & 3 & Escherichia coli $90.6 \%$ & Escherichia coli $99.9 \%$ \\
\hline 40 Chicken egg & 1 & Escherichia hermannii 96\% & Escherichia hermannii $97.7 \%$ \\
\hline $\begin{array}{l}41 \text { Canary leg necrosis, } \\
\text { human urine }\end{array}$ & 2 & $\begin{array}{l}\text { Klebsiella pneumoniae subsp. } \\
\text { pneumoniae } 94.5 \%\end{array}$ & $\begin{array}{l}\text { Klebsiella pneumoniae subsp. } \\
\text { pneumoniae } 98.2 \%\end{array}$ \\
\hline 42 Guinea pig & 1 & Morganella morganii 94\% & Morganella morganii $99.9 \%$ \\
\hline $\begin{array}{l}43 \text { Pork meat, animal feed, } \\
\text { chicken feces }\end{array}$ & 3 & Proteus mirabilis $92 \%$ & Proteus mirabilis $99.9 \%$ \\
\hline 44 Chicken feces & 1 & Providencia rettgeri $92 \%$ & $\begin{array}{l}\text { Providencia rettgeri } \\
\text { (no id } \% \text { ) }\end{array}$ \\
\hline 45 Chicken feces & 1 & Rahnella aquatilis $95 \%$ & Rahnella aquatilis \\
\hline
\end{tabular}




\begin{tabular}{|c|c|c|c|}
\hline & & & $($ no id $\%)$ \\
\hline $\begin{array}{l}46 \begin{array}{l}\text { Human skin, shampoo } \\
\text { sample }\end{array}\end{array}$ & 2 & Serratia marcescens $88.5 \%$ & $\begin{array}{l}\text { Serratia marcescens } 97.4 \% \\
\text { (no id } \% \text { for } 1 \text { strain) }\end{array}$ \\
\hline 47 Cow milk & 1 & Serratia liquefaciens $82 \%$ & Serratia liquefaciens $99.8 \%$ \\
\hline $\begin{array}{l}48 \text { Chicken liver and feces, } \\
\text { pigeon samples, guinea } \\
\text { pig bone marrow, animal } \\
\text { feed, wild boar liver, pig } \\
\text { samples }\end{array}$ & 21 & Salmonella spp.91.3\% & $\begin{array}{l}\text { Salmonella spp. } 91.1 \% \\
\text { (no id\% for } 8 \text { strains) }\end{array}$ \\
\hline 49 Chicken feces & 1 & Shigella sonnei $94 \%$ & Shigella sonnei $48.6 \%$ \\
\hline
\end{tabular}

Legend: a- ABIS \%id represents the percentage of similarity with taxa from the database, all databases containing a matrix where probabilistic incidence values are allocated for every taxon and their corresponding morpho-biochemical characters; b- apiweb ${ }^{\mathrm{TM}} \% \mathrm{id}$ is a probabilistic calculation using bioMerieux own system procedure.

Apiweb $^{\mathrm{TM}}$ returned an id\% lower than $80 \%$ for 9 species (Staphylococcus schleiferi, S. haemolyticus, Stenotrophomonas maltophilia, Salmonella spp., Shigella sonnei, Streptococcus pyogenes, Streptococcus bovis, Mannheimia haemolytica, and Lactobacillus fermentum), while ABIS returned low $\mathrm{id} \%$ for 2 species (Bacillus pumilus and Vibrio parahaemolyticus).

For the reference strain Clostridium tetani RO-2009 apiweb $^{\mathrm{TM}}$ returned Clostridium tetani without calculating an id\%, as an alternative result after Egerthella lenta $75 \%$, Clostridium spp. 16.9\% and Clostridium hystolyticum 3\%, while ABIS result was Clostridium tetani $97 \%$.

Proteus hauseri is biochemically similar to strains commonly identified as Proteus vulgaris, but esculin and salicin negative. Since these tests were not available neither in lab, nor in API galleries, the ATCC 13315 - Proteus hauseri strain was identified by ABIS as "P. vulgaris / P. hauseri", and by apiweb ${ }^{\mathrm{TM}}$ as "P. vulgaris group".

When identifying Salmonella, results were almost similar, the $\mathrm{id} \%$ differing only by $0.2 \%$. Moreover, 17 Salmonella strains were serologically confirmed by "O"antisera agglutination, 1 strain was confirmed by a national reference lab as Salmonella Typhimurium, and 1 strain was confirmed by a national reference lab as Salmonella Kottbus.

When identifying Mannheimia haemolytica, apiweb ${ }^{\mathrm{TM}}$ could not differentiate $M$. haemolytica from Pasteurella trehalosi. In one case ABIS returned Mannheimia glucosida as an alternative result. The validated result was $M$. haemolytica regarding ecology and pathogenicity.

When identifying Streptococcus pyogenes, for one strain apiweb $^{\mathrm{TM}}$ returned an alternative option: Streptococcus oralis, while ABIS returned Gemella palaticanis as an alternative. In both cases the final result was $S$. pyogenes regarding strain's ecology and pathogenicity.

When identifying Staphylococcus xylosus, ABIS returned multiple choices including: $S$. gallinarum, $S$. nepalensis and $S$. equorum. The validation of S. xylosus as final result is doubtful since apiweb ${ }^{\mathrm{TM}}$ database does not include the above taxa.

Comparison of the average $\mathrm{id} \%$ of wild strains per ABIS and apiweb ${ }^{\mathrm{TM}}$ databases shows that ABIS got $13.6 \%$ higher identification score for staphylococci $(93.5 \%$ vs $79.9 \%), 2.2 \%$ higher for streptococci $(90.7 \%$ vs $88.5 \%)$, $1.1 \%$ lower for Enterobacteriaceae (92.9\% vs 94.0\%) and $2.9 \%$ lower for other bacteria $(87.8 \%$ vs $90.7 \%)$. ABIS's average $\mathrm{id} \%$ of reference strains was $5.0 \%$ lower than apiweb's (93.9\% vs 98.9\%) (fig. 4).

Overall, ABIS achieved an average id $\%$ of $91.8 \%$ and apiweb $^{\text {TM }} 90.4 \%$, ABIS showing an identification percent higher by $1.4 \%$. Moreover, ABIS results have been correlated with ecological, morphological, biochemical and pathological characters of the identified taxa.

The variation range of apiweb ${ }^{\mathrm{TM}} \mathrm{id} \%$ (43.9 to $99 \%$ ) was wider than ABIS (77 to $99 \%$ ) which shows that the ABIS system is more homogenous and consistent.

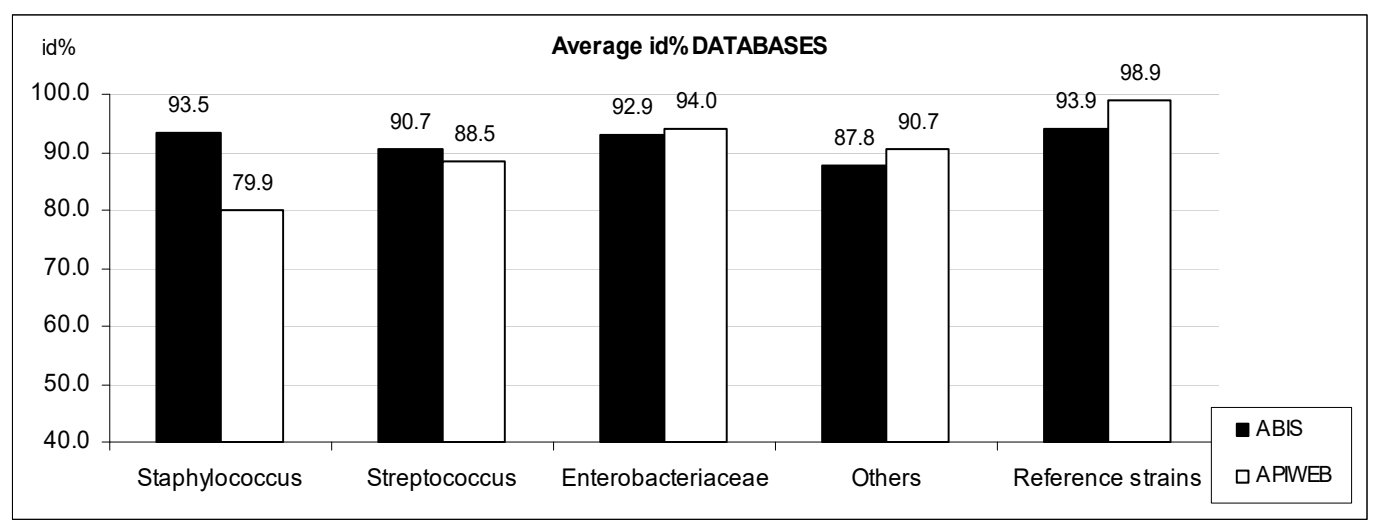

Figure 4. Graphic comparison between ABIS (id\%, black) and apiweb ${ }^{\mathrm{TM}}$ (id\%, white) database average $\mathrm{id} \%$ for wild and reference strains. 


\section{Conclusion}

Apiweb $^{\text {TM }}$ confirmed the results of ABIS with a high $\mathrm{id} \%$, proving very good taxa identification by ABIS program. ABIS online (ABIS online - bacterial identification software version 12, http://www.tgw1916.net) is capable of correct phenotypical species identification, generates reliable results, is a powerful tool for microbiology lab and the Encyclopedia connection provides essential information about the ecological significance, pathology and other features of the identified strains.

\section{References}

1. Borriello SP, Murray PR, Funke G (eds.). Topley \& Wilson's Microbiology \& Microbial Infections, Bacteriology, vol 2. Edward Arnold (Publishers) Ltd, London, 10th ed., 2006.

2. Buiuc D, Negut $M$ (eds). Tratat de microbiologie clinică. Editura Medicală, Bucureşti, ediția a III-a, 2009.

3. Gordon RE, Haynes WC, Pang CHN. The Genus Bacillus, Agriculture Handbook No.427. USDA, Washington DC, 1973.
4. Dworkin M, Falkow S, Rosenberg E, Schleifer KH (eds). The Prokaryotes, a handbook on the biology of bacteria, Vol. 4, Bacteria: Firmicutes, Cyanobacteria. Springer, New York, 3rd ed, 2006.

5. Buchanan RE, Gibbons NE (eds). Bergey's Manual of Determinative Bacteriology. Williams \& Wilkins, A Waverly Company, Baltimore, 8th ed., 1975.

6. Holt JG, Krieg NR, Sneath PHA, Staley JT et al. (eds). Bergey's Manual of Determinative Bacteriology. Williams \& Wilkins, A Waverly Company, Baltimore, 9th ed., 1994.

7. Vos PD, Garrity G, Jones D, Krieg NR et al. (eds). Bergey's Manual of Systematic Bacteriology, Vol. 3: The Firmicutes. Springer, New York, 2nd ed, 2009.

8. Goodfellow M, Kampfer P, Busse HJ, Trujillo et al. (eds). Bergey's Manual of Systematic Bacteriology, Vol. 5: The Actinobacteria, Part A. Springer, New York, 2nd ed., 2012.

9. Garrity GM (ed). Bergey's Manual of Systematic Bacteriology, Vol. 2: The Proteobacteria , Part B. Springer, New York, 2nd ed, 2004.

10. Funke G, von Graevenitz A, Clarridge III JE, Bernard KA. Clinical Microbiology of Coryneform Bacteria. Clin Microbiol Rev. 1997;10(1): 125-159. doi: 10.1128/CMR.10.1.12 